\title{
Calcification of coronary arteries and aortic valve and circulating a-klotho levels in patients with chronic kidney disease
}

\author{
Panagiotis Savvoulidis ${ }^{1,2}$, Andreas P. Kalogeropoulos ${ }^{3}$, Vasileios Raptis ${ }^{4}$, Vasileios Rafailidis ${ }^{5}$, Panagiotis \\ I. Georgianos ${ }^{4}$, Elias V. Balaskas ${ }^{4}$, Konstantinos Kouskouras ${ }^{5}$, Haralambos Karvounis ${ }^{2}$, Stavros \\ Hadjimiltiades $^{2}$
}

${ }^{1}$ Department of Cardiology, Royal Brompton \& Harefield NHS Foundation Trust, Sydney Street, London, UK; ${ }^{2}$ Department of Cardiology, AHEPA University Hospital, Aristotle University of Thessaloniki, Thessaloniki, Greece; ${ }^{3}$ Division of Cardiology, Department of Medicine, Stony Brook University, Stony Brook, NY, USA; ${ }^{4}$ Section of Nephrology and Hypertension, 1st Department of Medicine, AHEPA Hospital, ${ }^{5}$ Department of Radiology, AHEPA University General Hospital, Aristotle University of Thessaloniki, Thessaloniki, Greece

Contributions: (I) Conception and design: P Savvoulidis, AP Kalogeropoulos, V Raptis, S Hadjimiltiades; (II) Administrative support: P Savvoulidis, AP Kalogeropoulos, V Rafailidis, PI Georgianos, H Karvounis; (III) Provision of study materials or patients: P Savvoulidis, V Raptis, PI Georgianos, EV Balaskas, K Kouskouras; (IV) Collection and assembly of data: P Savvoulidis, AP Kalogeropoulos; (V) Data analysis and interpretation: P Savvoulidis, AP Kalogeropoulos; (VI) Manuscript writing: All authors; (VII) Final approval of manuscript: All authors.

Correspondence to: Andreas P. Kalogeropoulos, MD, MPH, PhD. Stony Brook University Medical Center, 101 Nicolls Road, Health Sciences Center, T-16, Rm 080, Stony Brook, NY 11794-8167, USA. Email: andreas.kalogeropoulos@stonybrook.edu.

Background: Evidence suggests that the anti-aging protein a-Klotho is a central modulator of mineral homeostasis. Circulating a-Klotho exerts endocrine activity and has been implicated in the process of vascular calcification, which is accelerated in patients with chronic kidney disease (CKD) and portends an unfavorable overall prognosis. However, the role of a-Klotho in this process remains unclear. The purpose of this study was to investigate the possible interaction between a-Klotho and the calcification of the aortic valve and coronary arteries in patients with CKD.

Methods: In this study we enrolled a total of 60 adult patients with CKD. Group 1 included 30 participants with CKD stage $V$ and group 2 included 30 participants with CKD stage III.

Results: Participants in group 1 had lower levels of circulating a-Klotho compared to group 2 (390; 280-590 vs. 722; 501-897 pg/mL; $\mathrm{P}=0.001)$, were of younger age $(55.5 ; 45-63$ vs. 69; 62-74 years; $\mathrm{P}<0.001)$, had lower body mass index $\left(25.6 ; 23.8-27.5\right.$ vs. $\left.28.2 ; 25.7-31.1 \mathrm{~kg} / \mathrm{m}^{2} ; \mathrm{P}=0.036\right)$, higher serum phosphate $(4.75 ; 4-5.6$ vs. 3.35; 2.9-3.8 mg/dL; $\mathrm{P}<0.001)$, higher calcium-phosphate product (41; 35.1-49.2 vs. 31.5; 28.6-35 $\mathrm{mg}^{2} / \mathrm{dL}^{2}$; $\mathrm{P}<0.001)$, and higher parathyroid hormone (PTH) levels (28.4; 15-44.6 vs. 7.05; 4.3-10.2 pmol /L; $\mathrm{P}<0.001)$.

Conclusions: No statistically significant difference was found between the two groups in terms of coronary arteries and aortic valve calcification. Calcitonin, PTH and phosphate were identified as predictors for circulating a-Klotho levels whereas, only hyperlipidemia was identified as predictor for coronary artery calcification. In conclusion, circulating a-Klotho is found to decrease with worsening CKD severity but no correlation was found between the levels of a-Klotho and severity of coronary arteries and aortic valve calcification.

Keywords: a-Klotho; vascular calcification; coronary artery calcification; aortic valve calcification; chronic kidney disease (CKD)

Submitted Sep 23, 2019. Accepted for publication Dec 12, 2019.

doi: $10.21037 /$ jtd.2020.01.49

View this article at: http://dx.doi.org/10.21037/jtd.2020.01.49 


\section{Introduction}

In 1997, Kuro-o et al. published their seminal study on a previously unknown gene, the mutation of which led to a clinical syndrome that resembled aging and encompassed growth retardation, short lifespan, arteriosclerosis, ectopic calcification, osteoporosis, skin atrophy and emphysema in mice homozygous for the transgene (1). Since the serendipitous discovery of this gene, ingeniously named Klotho after one of the three Fates in ancient Greek mythology (the Greek goddess who spins the thread of life) we have come a long way in defining its role and function although, several aspects of the physiology and pathophysiology still need to be further elucidated. The main transcript of this gene, a-Klotho, has been regarded as the anti-aging protein, particularly because the overexpression of this has been initially related to extended lifespan in mice (2). Of note, it was the discovery of Klotho that led to the identification of novel endocrine pathways and an unexpected link between aging and mineral metabolism. Mineral metabolism dysregulation and vascular calcification, a major finding in aging (3) and independent predictor of cardiovascular mortality and morbidity (4), are all hallmark characteristics in patients with chronic kidney disease (CKD), a well-established state of premature aging $(5,6)$. It was further documented that levels of circulating a-Klotho diminish with worsening renal function since early in the process (7). Therefore, it was suggested that a-Klotho plays a role in the process of vascular calcification in patients with CKD although, data are relatively scarce, equivocal and even conflicting $(8,9)$. In this study we sought to clinically explore the relationship between circulating a-Klotho levels and severity of calcification in the coronary arteries and the aortic valve in patients with distinctly different stages of CKD, namely between stage $\mathrm{V}$ (end-stage renal disease) and stage III CKD.

\section{Methods}

\section{Study protocol and measurements}

This was a cross-sectional study. We enrolled 60 adult patients with CKD who received care at AHEPA University Hospital, Thessaloniki, Greece, between January 01, 2016 and December 31, 2017. Patients with (I) active cancer; (II) systemic inflammatory or granulomatous disease; and (III) primary hyperparathyroidism were excluded. Group 1 included 30 patients with end-stage renal disease, CKD stage $\mathrm{V}\left(\mathrm{eGFR}<15 \mathrm{~mL} / \mathrm{min} / 1.73 \mathrm{~m}^{2}\right)$ under renal replacement therapy in the form of regular intermittent hemodialysis. Group 2 included 30 outpatients (OP) with CKD stage III $\left(\mathrm{eGFR} \geq 30,<60 \mathrm{~mL} / \mathrm{min} / 1.73 \mathrm{~m}^{2}\right)$ with stable eGFR for the last at least 3 months prior to enrollment. For the calculation of eGFR the 4-variable, abbreviated modification of diet in renal disease (MDRD) study equation $\left(\mathrm{eGFR}=186 \times\right.$ serum creatinine $\mathrm{e}^{-1.154} \times$ age $^{-0.203}$ $\times 0.742$ if female) was used $(10,11)$.

Clinical data were collected through the primary physician, the Hospital Medical Records and, by directly interviewing the patients. Baseline demographic and clinical data were recorded as follows: age, gender, primary kidney disease, body mass index, blood pressure, medication history, and comorbid diseases. For laboratory measurements, blood samples were drawn in the morning after an overnight fast of at least 8 hours for the OP group. For all patients in the CKD-V group, dry weight was used as reference weight. Blood samples were drawn at the beginning of the hemodialysis session and, the hemodialysis session in the middle of the week was selected for the study purposes.

\section{Laboratory testing}

After centrifuging whole blood at 3,000 rpm for 10 minutes at $4{ }^{\circ} \mathrm{C}$, supernatant serum was immediately stored in aliquots in deep freeze $\left(-80^{\circ} \mathrm{C}\right)$ for future analysis. No freeze-thaw-freeze cycle of the stored serum samples occurred at any point of the study. Human soluble a-Klotho was measured using a commercially available for research use, solid phase sandwich enzyme-linked immunosorbent assay (ELISA) kit (IBL International GmbH, Hamburg, Germany). It has been previously tested, and the intra-assay and inter-assay coefficient of variation (CV) were $<10 \%(12)$. The serum soluble $\alpha$-Klotho levels with this assay range between 93.75 and $6,000 \mathrm{pg} / \mathrm{mL}$, cross reactivity with human a-Klotho is $100 \%$ (specificity) and sensitivity is as low as $6.15 \mathrm{pg} / \mathrm{mL}$. Serum parathyroid hormone (PTH) was assayed by a double ('sandwich') electro-chemiluminescence immunoassay (Roche Diagnostics GmbH, Mannheim, Germany); intra-assay and inter-assay $\mathrm{CV}$ are $<10 \%$ and serum PTH levels with this assay range between 5.50 and 2,300 pg/mL. Serum 25 hydroxyvitamin D [25 (OH) D] was analyzed with the radioimmunoassay method (DIAsource ImmunoAssays S.A., Belgium); intra-assay and inter-assay $\mathrm{CV}$ are $<10 \%$ and detection limit is $1.5 \mathrm{ng} / \mathrm{mL}$. Serum calcitonin was analyzed by a double ('sandwich') immunoradiometric assay (Institute of Isotopes Ltd., Budapest, Hungary); intra-assay and inter-assay $\mathrm{CV}$ are 
$<10 \%$, serum calcitonin levels with this assay range between 0 and $2,000 \mathrm{pg} / \mathrm{mL}$ and sensitivity is $1.5 \mathrm{pg} / \mathrm{mL}$.

\section{Imaging process}

All patients had a non-contrast cardiac multi-slice computed tomography (MSCT) using a 128-slice scanner (CT Optima 660, GE Healthcare) on the day or within a week of the blood samples draw. Agatston score was used for the quantitative evaluation of the degree of calcification of the coronary arteries and aortic valve (13). Prospective electrocardiogram triggering ( $75 \%$ of $\mathrm{R}-\mathrm{R}$ interval) was used with a slice thickness of $2.5 \mathrm{~mm}$. The scan range extended from the level of clavicles to the diaphragm. The following scanning parameters were applied: collimation width: $32 \times 0.625 \mathrm{~mm}$; rotation time: $330 \mathrm{~ms}$; tube voltage: $120 \mathrm{kV}$; and maximum effective tube current: $64 \mathrm{~mA}$. Image reconstruction was gated retrospectively to $75 \%$ of $\mathrm{R}-\mathrm{R}$ interval. MSCT images were reconstructed using a cardiac standard filter with a slice thickness of $2.5 \mathrm{~mm}$. The slice thickness of the images reconstructed and reviewed was $0.625 \mathrm{~mm}$ for optimal isotropic imaging. MSCT data sets were transferred to an offline workstation (AW workstation, GE Healthcare) for image analysis. Aortic valve calcium score and coronary arteries calcium score were determined 2-dimensionally by using the calcium score data sets on the workstation and, defined by Agatston units (AU). The SmartScore 4.0 (General Electric Company, France) software package was used for the quantification of Agatston score of the coronary arteries and the aortic valve. The aortic valve calcium score area of interest comprised the valve cusps and aortic annulus. Total coronary calcium score was determined by summing the individual lesion scores of each of the main coronary arteries. All MSCT images were analyzed by 2 independent, appropriately trained and experienced physicians. In the case of different observer readings, a consensus reading was performed and used in the final analysis.

\section{Statistical analysis}

We described continuous variables as median (25th-75th percentile) and categorical variables as $\mathrm{N}(\%)$. We compared variables between groups using the nonparametric MannWhitney U test for continuous and Fisher's exact test for categorical variables. We examined bivariate correlations of a-Klotho levels with clinical variables and calcification scores using the Spearman correlation coefficient. To identify determinants of a-Klotho levels, we log-transformed
a-Klotho levels because of lognormal distribution and used stepwise linear regression with probability to remove the variable $>0.1$. To identify determinants of coronary and aortic calcification, we log-transformed the corresponding calcium scores, assuming a value of 0 (log-transformed 1 ) for absent calcium and, used stepwise linear regression as above. Because of the small sample size, all regression estimates, and confidence intervals were calculated with resampling (jackknife) to ensure robust variable selection and stable estimates. STATA 14.2 (StataCorp LP, College Station, TX, USA) software package was used for all analyses.

The study adhered to the tenets of the declaration of Helsinki, as revised in 2013, and it was approved by the Ethics Committee of AHEPA University Hospital, Aristotle University of Thessaloniki. All patients provided written informed consent prior to enrollment. The study is registered on ClinicalTrials.gov (Identification number NCT03858413).

\section{Results}

We identified 31 patients with $C K D-V$ under intermittent hemodialysis and 32 outpatients with stable CKD-III. In total, 63 patients enrolled to the study with 60 of them participating as 2 of the patients from the first group and 1 from the second group withdrew consent. Baseline clinical and biochemical characteristics are presented in Table 1. Renal function, as reflected by eGFR, in our whole population averaged $23.9 \mathrm{~mL} / \mathrm{min} / 1.73 \mathrm{~m}^{2}$ (median: 19.6; $25^{\text {th }}-75^{\text {th }}$ percentile: $5.9-41$ ) and included patients with CKD stage $\mathrm{V}(\mathrm{N}=30)$, median eGFR $5.9 \mathrm{~mL} / \mathrm{min} / 1.73 \mathrm{~m}^{2}$ (5.1-7.6), and CKD stage III $(\mathrm{N}=30)$, median eGFR $41 \mathrm{~mL} / \mathrm{min} / 1.73 \mathrm{~m}^{2}$ (35.4-45). Forty-two patients (70\%) were males (18 with CKD-III and 24 with CKD-V). Mean age in the CKD-III group was 68.2 years $(69 ; 65-74$ years) and mean age in the CKD-V group was 54.6 years $(55.5 ; 45-63$ years); there was statistically significant difference between the two groups with overall, younger patients in the $\mathrm{CKD}-\mathrm{V}$ group $(\mathrm{P}<0.001)$.

Serum a-Klotho was significantly lower in the CKD-V group compared to the CKD-III group (390; 280-590 vs. 722; 501-897 pg/mL, respectively; $\mathrm{P}=0.001$ ) (Figure 1).

In a multivariate analysis including clinical and laboratory covariates, serum calcitonin, FT3 and FT4 were independently associated with serum a-Klotho (Table 2). In bivariate analysis, serum a-Klotho was negatively correlated with calcitonin $(\mathrm{P}=-0.508, \mathrm{P}<0.001)$, PTH $(\mathrm{P}=-0.310$, $\mathrm{P}=0.016)$ and phosphate $(\mathrm{P}=-0.302, \mathrm{P}=0.019)$ (Figure 2). No correlation was found between a-Klotho levels and calcium score in the coronary arteries or the aortic valve. 
Table 1 Patient characteristics in the total studies population and of group 1 (CKD-V) in comparison to group 2 (CKD-III)

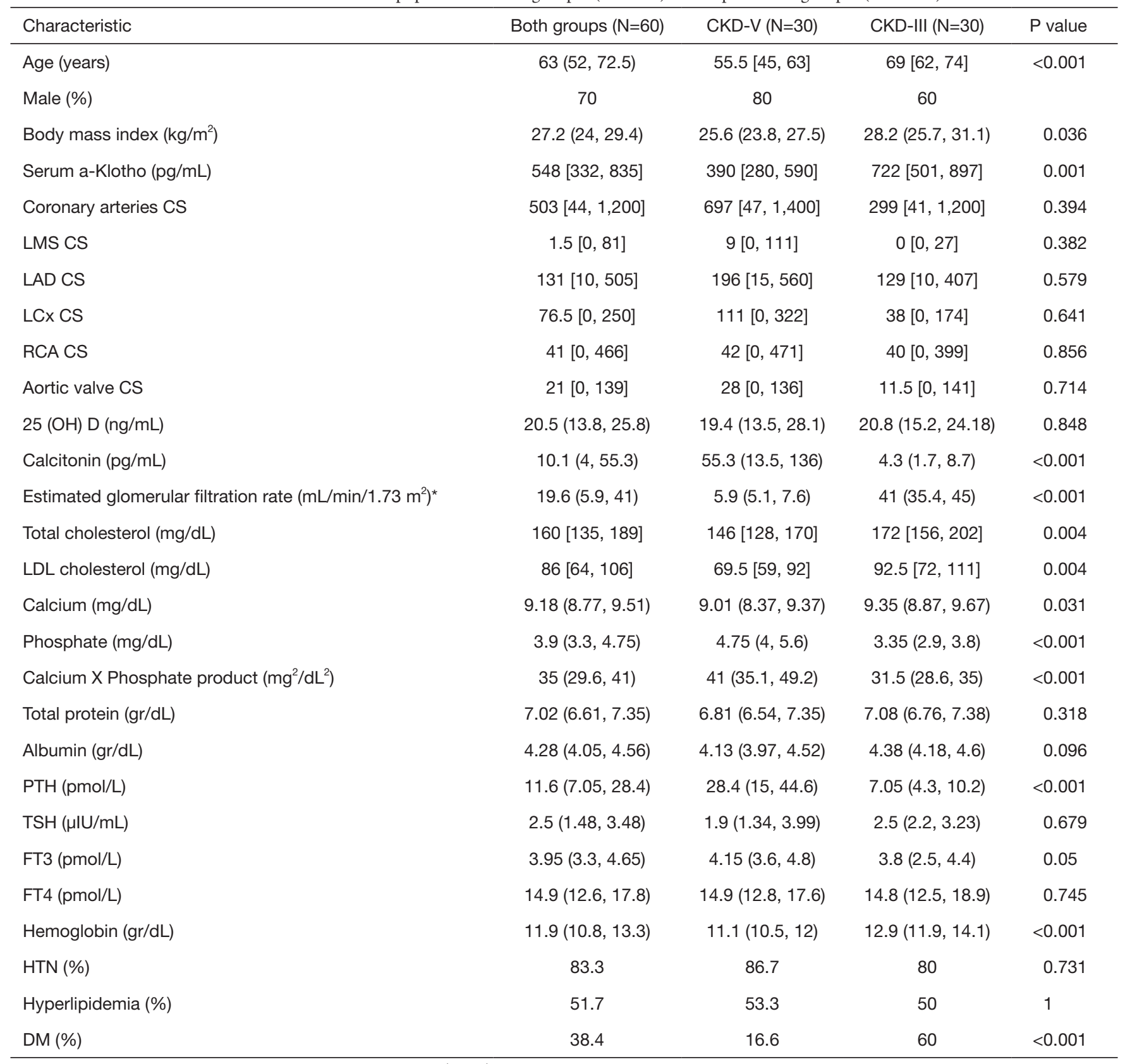

Values for continuous variables represent median $\left(25^{\text {th }}, 75^{\text {th }}\right.$ percentile). *, calculated with the Modification of Diet in Renal Disease study equation. CS, calcium score; LMS, left main stem; LAD, left anterior descending artery; LCx, left circumflex artery; RCA, right coronary artery; $25(\mathrm{OH})$ D, 25 hydroxyvitamin D; PTH, parathyroid hormone; TSH, thyroid-stimulating hormone; FT3, free triiodothyronine; FT4, thyroxine; HTN, hypertension; DM, diabetes mellitus.

No statistically significant difference was found in calcium score in coronary arteries between the two groups (697; 47-1,400 vs. 299; 41-1,200; $\mathrm{P}=0.394)$. The same applied when we broke down the comparisons into persegment measurements (Table 1). There was no statistically significant difference between the two groups in aortic valve calcium score (28; 0-136 vs. 11.5; 0-141; $\mathrm{P}=0.714$ ) (Table 1).

In a multivariate analysis performed to identify associations of calcium score in the coronary arteries and aortic valve among clinical characteristics and biomarkers, the 


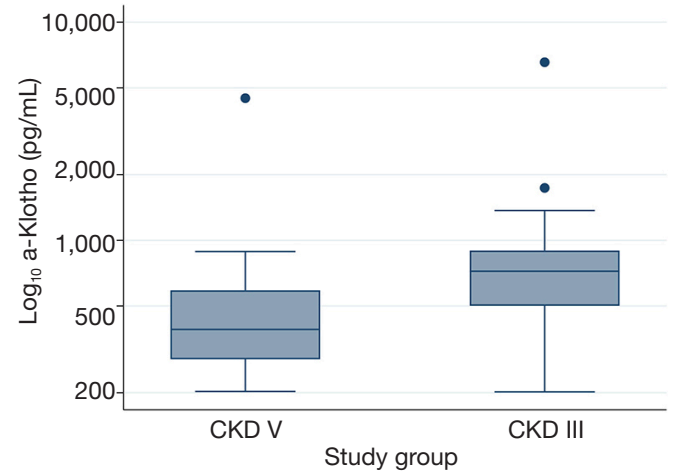

Figure 1 Serum a-Klotho levels in group 1 (CKD-V) in comparison to group 2 (CKD-III).

Table 2 Multivariate analysis performed to identify predictors of serum a-Klotho

\begin{tabular}{lccc}
\hline Variable & Coefficient & $\begin{array}{c}95 \% \text { confidence } \\
\text { intervals }\end{array}$ & $P$ \\
\hline Log-Calcitonin, pg/mL & -0.215 & $-0.313,-0.118$ & $<0.001$ \\
FT3, pmol/L & 0.036 & $0.002,0.070$ & 0.037 \\
FT4, pmol/L & 0.188 & $0.054,0.323$ & 0.007
\end{tabular}

only variable correlated with calcium score in the coronary arteries was the presence of hyperlipidemia (Table 3).

\section{Discussion}

In this observational study including 60 patients with CKD, we describe the association between circulating a-Klotho levels and calcification in the coronary arteries and aortic valve. Circulating a-Klotho levels were significantly lower in the CKD-V group compared to the CKD-III group in our cohort. However, no statistically significant difference was noted between the two groups in the coronary arteries or aortic valve calcification. Calcitonin, PTH and phosphate were associated with circulating a-Klotho levels. Hyperlipidemia was correlated with coronary artery calcification whereas no variable was associated with aortic valve calcification in the studied population. Therefore, no correlation was suggested between the levels of circulating a-Klotho and calcification in the coronary arteries and aortic valve, as expressed in Agatston score units.

Klotho is part of the FGF-23/Klotho, 1,25-dihydroxyvitamin D or calcitriol $\left[1,25(\mathrm{OH})_{2} \mathrm{D}\right]$ and PTH axis and have a central role in regulating the mineral homeostasis, namely, regulate
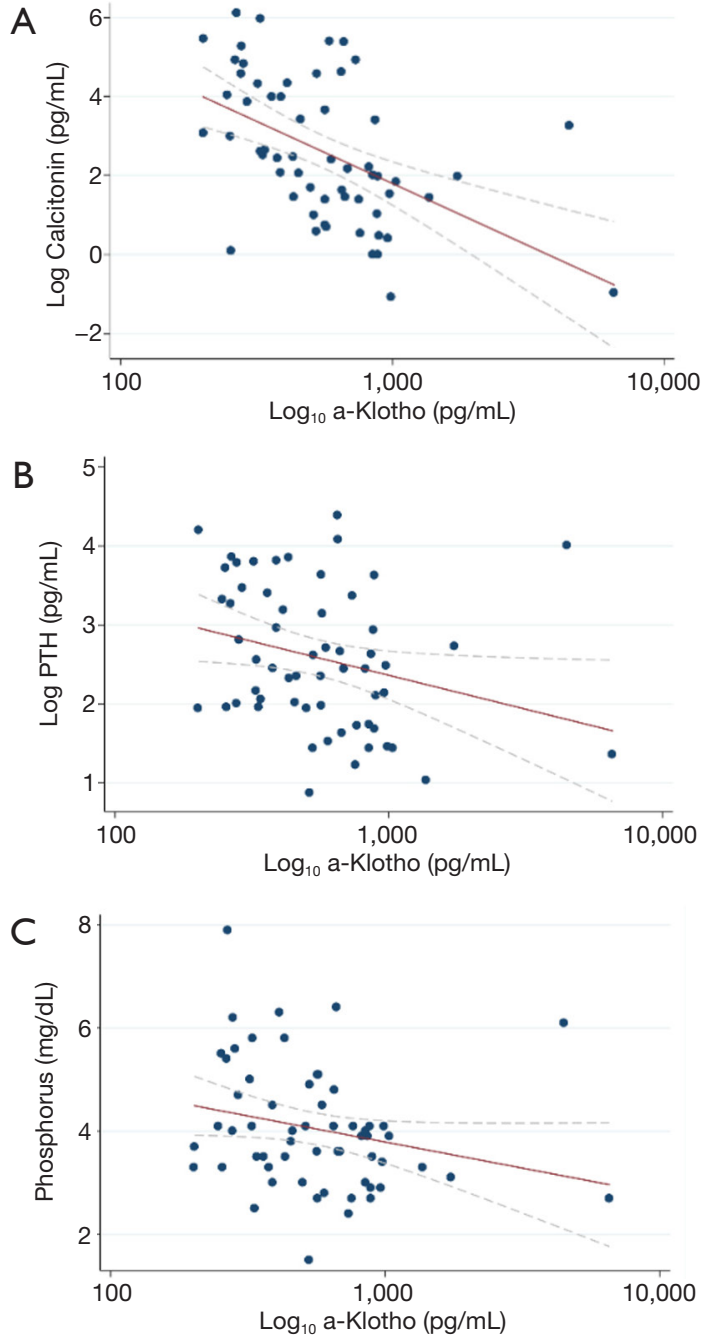

Figure 2 Correlation tests of serum a-Klotho with: (A) Calcitonin, (B) PTH and (C) Phosphat.

Table 3 Multivariate analysis performed to identify predictors of calcium score in the coronary arteries

\begin{tabular}{lccc}
\hline Variable & Coefficient & $95 \%$ confidence intervals & $P$ \\
\hline Hyperlipidemia* $^{*} 0.624$ & $0.027,1.222$ & 0.041 \\
\hline *, defined as LDL cholesterol & $\geq 190 \mathrm{mg} / \mathrm{dL}$ without diabetes \\
mellitus or LDL cholesterol & $70-189 \mathrm{mg} / \mathrm{dL}$ with concomitant \\
diabetes mellitus or already on lipid-lowering treatment.
\end{tabular}

the calcium and phosphate concentration within very strict and narrow ranges and in a very delicate balance to commensurate with the body demands. The main type of Klotho is a-Klotho, a type I, single-pass transmembrane protein of 1,012 amino acids in humans (1). Membrane Klotho is an obligatory co- 
factor for FGF-23, a 251-amino acid hormone predominantly produced from osteocytes and osteoblasts during active bone remodeling (14), and increase urinary phosphate excretion and suppresses synthesis of $1,25(\mathrm{OH})_{2} \mathrm{D}$. Apart from the membrane form of a-Klotho, there is the circulating form which is a product of shedding from proteases and secretases $(15,16)$. This circulating form has been shown to have hormonal properties independent from FGF-23.

There is mounting evidence that vascular calcification is an independent predictor of cardiovascular morbidity and mortality in CKD (17-19). It is suggested that circulating a-Klotho is pivotal in the process of vascular calcification because it (I) regulates phosphate levels (20) and (II) protects against vascular calcification by inactivating the FGFR1/ERK signaling pathway in bone marrow-derived mesenchymal stem cells (21). Decreased levels of circulating serum Klotho have been associated with increased arterial stiffness (7).

This current study confirms previous knowledge that circulating a-Klotho levels deplete as renal function declines (22-25). Furthermore, a-Klotho levels in the studied population were associated with phosphate, PTH and calcitonin which is in accordance with previous studies (23). a-Klotho has a central role in the regulation of mineral homeostasis and hence, phosphate levels (as a result of the complex regulatory axis), PTH (as a regulatory hormone for calcium and phosphate levels homeostasis) and calcitonin (as a regulatory hormone for calcium levels homeostasis) were associated with circulating a-Klotho levels.

By contrast, there was a statistically non-significant difference in the calcification severity between the two groups. This could be postulated to be explained by the severity of CKD in the CKD-III group as, the majority of the participants were in stage IIIb CKD (eGFR $\geq 30,<45 \mathrm{~mL} /$ $\min / 1.73 \mathrm{~m}^{2}$ ), namely, renal function in those participants was moderately to severely impaired rather than mildly to moderately impaired and closer to CKD IV and V stages.

Our study has several limitations. This was a crosssectional study and as such, no temporal causation can be safely inferred. As patients were randomly selected, the significant difference in age between the two groups may account to some extent for the non-significant difference in calcification severity in the coronary arteries and the aortic valve. The subgroup of CKD stage $\mathrm{V}$ included younger patients and, the calcification severity was not different compared to patients with CKD stage III. It could be postulated that if patients were age-matched there could have been a significant difference in the extent of calcification. We believe that future studies trying to shed more light on the differential severity in calcification should include more patients to enhance power and a more diverse CKD cohort in an effort to include participants within all different stages of CKD severity. In addition, longterm follow-up of these patients would provide evidence of temporal association between circulating a-Klotho levels and calcification in the heart vasculature. Future studies should age-match the population of the sub-groups to exclude age as a confounding factor. Furthermore, all participants were of Caucasian ancestry secondary to the race distribution at the site of the study. Therefore, no conclusions can be drawn for patients with CKD of Black or other race.

In conclusion, circulating a-Klotho is found to decrease with worsening CKD severity but no correlation was found between the levels of a-Klotho and severity of coronary arteries and aortic valve calcification.

\section{Acknowledgments}

Funding: None.

\section{Footnote}

Conflicts of Interest: The authors have no conflicts of interest to declare.

Ethical Statement: The authors are accountable for all aspects of the work in ensuring that questions related to the accuracy or integrity of any part of the work are appropriately investigated and resolved. All procedures performed in this study were in accordance with the ethical standards of the institutional and national research committee (ClinicalTrials. gov Identification number NCT03858413) and with the Helsinki Declaration as revised in 2013.

Open Access Statement: This is an Open Access article distributed in accordance with the Creative Commons Attribution-NonCommercial-NoDerivs 4.0 International License (CC BY-NC-ND 4.0), which permits the noncommercial replication and distribution of the article with the strict proviso that no changes or edits are made and the original work is properly cited (including links to both the formal publication through the relevant DOI and the license). See: https://creativecommons.org/licenses/by-nc-nd/4.0/.

\section{References}

1. Kuro-o M, Matsumura Y, Aizawa H, et al. Mutation of the 
mouse klotho gene leads to a syndrome resembling ageing. Nature 1997;390:45-51.

2. Kurosu H, Yamamoto M, Clark JD, et al. Suppression of aging in mice by the hormone Klotho. Science 2005;309:1829-33.

3. Tesauro M, Mauriello A, Rovella V, et al. Arterial ageing: from endothelial dysfunction to vascular calcification. J Intern Med 2017;281:471-82.

4. Cannata-Andia JB, Rodriguez-Garcia M, CarrilloLopez N, et al. Vascular calcifications: pathogenesis, management, and impact on clinical outcomes. J Am Soc Nephrol 2006;17:S267-73.

5. Palit $\mathrm{S}$, Kendrick J. Vascular calcification in chronic kidney disease: role of disordered mineral metabolism. Curr Pharm Des 2014;20:5829-33.

6. Kooman JP, Kotanko P, Schols AM, et al. Chronic kidney disease and premature ageing. Nat Rev Nephrol 2014;10:732-42.

7. Kitagawa $M$, Sugiyama $\mathrm{H}$, Morinaga $\mathrm{H}$, et al. A decreased level of serum soluble Klotho is an independent biomarker associated with arterial stiffness in patients with chronic kidney disease. PLoS One 2013;8:e56695.

8. Nattero-Chavez L, Luque-Ramirez M, Moncayo S, et al. Circulating soluble klotho is not associated with an elevated ankle-brachial index as a surrogate marker of early arterial calcification in patients with type 1 diabetes mellitus and no evidence of renal dysfunction. Diabetes Metab 2019;45:589-92.

9. Vervloet MG, Adema AY, Larsson TE, et al. The role of klotho on vascular calcification and endothelial function in chronic kidney disease. Semin Nephrol 2014;34:578-85.

10. Levey AS, Bosch JP, Lewis JB, et al. A more accurate method to estimate glomerular filtration rate from serum creatinine: a new prediction equation. Modification of Diet in Renal Disease Study Group. Ann Intern Med 1999;130:461-70.

11. Levey AS, Coresh J, Greene T, et al. Expressing the Modification of Diet in Renal Disease Study equation for estimating glomerular filtration rate with standardized serum creatinine values. Clin Chem 2007;53:766-72.

12. Heijboer AC, Blankenstein MA, Hoenderop J, et al. Laboratory aspects of circulating alpha-Klotho. Nephrol Dial Transplant 2013;28:2283-7.

13. Agatston AS, Janowitz WR, Hildner FJ, et al. Quantification of coronary artery calcium using ultrafast computed tomography. J Am Coll Cardiol 1990;15:827-32.

14. Shimada T, Mizutani S, Muto T, et al. Cloning and characterization of FGF23 as a causative factor of tumor-induced osteomalacia. Proc Natl Acad Sci U S A 2001;98:6500-5.

15. Chen CD, Podvin S, Gillespie E, et al. Insulin stimulates the cleavage and release of the extracellular domain of Klotho by ADAM10 and ADAM17. Proc Natl Acad Sci U S A 2007;104:19796-801.

16. Xu Y, Sun Z. Molecular basis of Klotho: from gene to function in aging. Endocr Rev 2015;36:174-93.

17. Fox CS, Larson MG, Vasan RS, et al. Cross-sectional association of kidney function with valvular and annular calcification: the Framingham heart study. J Am Soc Nephrol 2006;17:521-7.

18. Goodman WG, Goldin J, Kuizon BD, et al. Coronaryartery calcification in young adults with end-stage renal disease who are undergoing dialysis. $\mathrm{N}$ Engl J Med 2000;342:1478-83.

19. Blacher J, Guerin AP, Pannier B, et al. Arterial calcifications, arterial stiffness, and cardiovascular risk in end-stage renal disease. Hypertension 2001;38:938-42.

20. Hu MC, Shi M, Zhang J, et al. Klotho deficiency causes vascular calcification in chronic kidney disease. J Am Soc Nephrol 2011;22:124-36.

21. Zhang W, Xue D, Hu D, et al. Secreted klotho protein attenuates osteogenic differentiation of human bone marrow mesenchymal stem cells in vitro via inactivation of the FGFR1/ERK signaling pathway. Growth Factors 2015;33:356-65.

22. Neyra JA, Hu MC. alphaKlotho and Chronic Kidney Disease. Vitam Horm 2016;101:257-310.

23. Rotondi S, Pasquali M, Tartaglione L, et al. Soluble alpha -Klotho Serum Levels in Chronic Kidney Disease. Int J Endocrinol 2015;2015:872193.

24. Pavik I, Jaeger P, Ebner L, et al. Secreted Klotho and FGF23 in chronic kidney disease Stage 1 to 5: a sequence suggested from a cross-sectional study. Nephrol Dial Transplant 2013;28:352-9.

25. Kim HR, Nam BY, Kim DW, et al. Circulating alphaklotho levels in CKD and relationship to progression. Am J Kidney Dis 2013;61:899-909.

Cite this article as: Savvoulidis P, Kalogeropoulos AP, Raptis V, Rafailidis V, Georgianos PI, Balaskas EV, Kouskouras K, Karvounis H, Hadjimiltiades S. Calcification of coronary arteries and aortic valve and circulating a-klotho levels in patients with chronic kidney disease. J Thorac Dis 2020;12(3):431437. doi: $10.21037 /$ jtd.2020.01.49 